\title{
La peau, frontière perméable du corps ?
}

Die Haut: eine durchlässige Körpergrenze?

The Skin, a Permeable Corporeal Border?

\section{Florence Bancaud}

\section{(2) OpenEdition}

\section{Journals}

Édition électronique

URL : https://journals.openedition.org/ceg/9817

DOI : $10.4000 /$ ceg. 9817

ISSN : 2605-8359

\section{Éditeur}

Presses Universitaires de Provence

\section{Édition imprimée}

Date de publication : 20 janvier 2020

Pagination : 127-140

ISBN : 979-10-320-0253-7

ISSN : 0751-4239

\section{Référence électronique}

Florence Bancaud, «La peau, frontière perméable du corps ? », Cahiers d'Études Germaniques [En ligne], 78 | 2020, mis en ligne le 28 octobre 2021, consulté le 31 janvier 2022. URL : http:// journals.openedition.org/ceg/9817; DOI : https://doi.org/10.4000/ceg.9817 


\title{
La peau, frontière perméable du corps?
}

Florence BANCAUD

Aix-Marseille Université, ÉCHANGES (EA 4236), Aix-en-Provence

Ce qu'il y a de plus profond en l'homme, c'est la peau.

Paul Valéry ${ }^{1}$

La peau est non seulement l'enveloppe de l'organisme, elle en est aussi le miroir et le résumé. Jean-Paul Escande ${ }^{2}$

\begin{abstract}
Was für ein Schauplatz: die Haut. Und was für ein Theater um sie gemacht wird: um die empfindende und lesbare Fläche zwischen Welt und Selbst. Ein Austragungsort, ein Spiegel, auch ein Dementi nicht nur der einzelnen Biografie, sondern der Geschichte von Kultur und Natur. Eine Schnittstelle, buchstäblich, von Wissenschaft und Kunst, von Erfahrung und Erkenntnis, von Sprache und Fühlen, von Intimität und Öffentlichkeit. Mit der Haut wenden wir uns an die Welt, an der Haut behelligt und berührt uns die Welt, als „Dickhäuter“, wie Büchners Danton sagt: „... wir reiben nur das grobe Leder aneinander $\mathrm{ab}$, - wir sind sehr einsam“" 3 .
\end{abstract}

Qu'il soit exposé aux caresses, aux coups, aux blessures, aux atteintes de l'âge ou de la maladie, le corps reste la surface de contact première avec l'altérité, que cette dernière soit bienfaisante ou malfaisante. Frontière du corps-frontière, limite entre le moi et le monde ${ }^{4}$, le corps vécu de l'intérieur (Leib) et éprouvé ou perceptible du dehors (Körper), l'organisme et le milieu, la peau, indépassable " mur du corps ${ }^{5}$ " est cette enveloppe, ce lien tissé avec le monde dont Didier

1. Paul Valéry, L'Idée fixe, Euvres II, Paris, Gallimard (coll. " La Pléiade »), 1960, p. 215-216.

2. Jean-Paul Escande, cité par Paul Dagognet, La Peau découverte, Paris, Les Empêcheurs de penser en rond, 1993, p. 11.

3. Elisabeth von Thadden, "Ich, von außen. Claudia Benthien untersucht die Haut ", Die Zeit, 25. November 1999. Voir le livre de Claudia Benthien : Haut : Literaturgeschichte - Körperbilder - Grenzdiskurse, Reinbek, Rowohlt Taschenbuch Verlag, 1999.

4. Oliver Jahraus, Die Aktion des Wiener Aktionismus. Subversion der Kultur und Dispositionierung des Bewußtseins, München, Fink, 2000, p. 212 : " [Die Haut] erscheint landläufig vor allem als Grenze des Selbst; sie beherbergt es und schließt es nach außen ab. "

5. Sylvia Plath, Journaux, Euvres, édition établie et présentée par Patricia Godi, Paris, Gallimard (coll. " Quarto "), 2011. 
Anzieu rappelle qu'elle est une " donnée originaire à la fois d'ordre organique et d'ordre imaginaire, comme système de protection de notre individualité en même temps que comme premier instrument et lieu d'échange avec autrui ", principe de " différenciation interne et principe de contenance ${ }^{6}$ ". De fait, la peau, plus qu'un organe, est " un ensemble d'organes différents [...]. De tous les organes des sens, c'est le plus vital " "; on peut vivre aveugle ou sourd, mais on ne survit pas sans la majeure partie de la peau qui pèse $20 \%$ du poids total du corps d'un nouveau-né et $18 \%$ de celui d'un adulte ${ }^{8}$; système de plusieurs organes de sens (toucher, pression, douleur, chaleur), elle reste en étroite connexion avec les autres organes des sens externes (ouie, vue, odorat, goût) et les sensibilités kinésiques et d'équilibration; elle combine les dimensions spatiale et temporelle, évaluant l'espace et le temps sur sa surface. Enfin, elle réagit à divers stimuli, restant " presque toujours disponible pour recevoir des signes, apprendre des codes, sans qu'ils interfèrent avec d'autres ${ }^{9}$. " La peau constitue donc un des noyaux les plus centraux de notre être. Elle " respire et perspire, sécrète et élimine, entretient le tonus, stimule la respiration, la circulation, la digestion, l'excrétion et la reproduction ${ }^{10} "$; maintenant le corps autour du squelette et de sa verticalité, elle protège (par sa couche cornée superficielle, son vernis de kératine, son coussinet de graisse) contre les agressions extérieures, capte et transmet des excitations ou informations utiles.

[La peau] soustrait l'équilibre de notre milieu interne aux perturbations exogènes, mais aussi dans sa forme, sa texture, sa coloration, ses cicatrices, elle conserve les marques de ces perturbations. À son tour, cet état intérieur qu'elle est censée préserver, elle le révèle en grande partie au-dehors; elle est aux yeux des autres un reflet de notre bonne ou mauvaise santé organique et un miroir de notre âme ${ }^{11}$.

Bien plus qu'une simple enveloppe du sujet, la peau contribue à forger son identité et à préserver ou non son intégrité. Anzieu va jusqu'à parler de " moipeau ", désignant par là " une figuration dont le Moi de l'enfant se sert au cours des phases précoces de son développement pour se représenter lui-même comme Moi contenant les contenus psychiques, à partir de son expérience de la surface du corps ${ }^{12}$. "Ce moi-peau est originellement le sac qui contient et retient à l'intérieur le bon et le plein que l'allaitement, les soins, le bain de paroles y ont accumulés ; c'est ensuite

l'interface qui marque la limite avec le dehors et maintient celui-ci à l'extérieur, c'est la barrière qui protège de la pénétration par les avidités et les agressions en provenance des autres êtres ou objets. C'est un lieu et un moyen primaire de communication avec

6. Didier Anzieu, Le Moi-peau, Paris, Dunod, 2006, p. 25 et p. 27.

7. Ibid., p. 36. La peau pèse 3 kilos pour un adulte d'un poids total de $70 \mathrm{~kg} ; 1 \mathrm{~cm}^{2}$ contiendrait 3 vaisseaux sanguins, 10 poils, 12 nerfs, 15 glandes sébacées, 100 sudoripares, 3 millions de cellules.

8. Ibid., p. 35-36.

9. Ibid., p. 36.

10. Ibid., p. 36-37.

11. Ibid., p. 39.

12. Ibid., p. 61. 
autrui, d'établissement de relations signifiantes [...] et une surface d'inscription des traces laissées par ceux-ci ${ }^{13}$.

Anzieu conclut à huit fonctions essentielles du moi-peau ${ }^{14}$ : une fonction de maintenance du psychisme, une fonction contenante, une fonction de pareexcitation, une fonction d'individuation du soi ; une fonction d' "intersensorialité " prévenant l'angoisse de morcellement du corps, une fonction de soutien de l'excitation sexuelle, de recharge libidinale et enfin d'inscription des traces sensorielles tactiles.

Mais rappelons aussi, avec François Dagognet, que la peau, loin d'être une simple " cloison défensive ${ }^{15}$ ", est un indice majeur du travail du temps sur le corps humain : elle dissimule en elle à la fois un processus de mort (la kératinisation, la suppression des noyaux dans les cellules de plus en plus aplaties) ainsi qu'un dynamisme de constant renouvellement : elle subit donc une altération perpétuelle en même temps qu'elle est appelée à la perpétuation.

Pourtant, nous ne percevons notre peau que dans le contact avec le monde des objets et le monde humain : elle est en effet lieu de la caresse, de la piqûre, de la griffure, de la plaie, du coup, de la cicatrice, du tatouage, de l'empreinte $\mathrm{du}$ réel sur le corps ${ }^{16}$. Nous ne percevons donc distinctement la surface de notre peau que quand nous sommes en contact avec la réalité et ses objets qui éprouvent les limites entre le Körper, corps objectif, perçu du dehors et le corps vécu du dedans, le Leib. De même, le moi conscient est avant tout un moi-corps pour Freud, selon qui le tactile fournit à la fois une perception externe et une perception interne : je sens l'objet qui touche ma peau en même temps que je sens ma peau touchée par l'objet.

La peau est donc emplie de paradoxes,

perméable et imperméable [...], superficielle et profonde. Elle est véridique et trompeuse [...]. Elle matérialise par sa nudité notre dénuement, mais aussi notre excitation sexuelle. Elle traduit par sa minceur, sa vulnérabilité, notre détresse originaire, plus grande que celle de toutes les autres espèces, et en même temps notre souplesse adaptative et évolutive. Elle sépare et unit les différentes sensorialités. Elle a [...] un statut d'intermédiaire, d'entre-deux, de transitionnalité ${ }^{17}$.

C'est donc sa fonction d'individuation et de lien social qui fait d'elle tant une délimitation du moi qu'un lien entre moi et autrui. Nous proposons ici de de l'explorer en dépassant l'opposition entre superficialité et profondeur et en partant du postulat que c'est à la surface de la peau, dans son contact avec l'autre et le monde que se révèle la profondeur de l'être et du sens :

Prendre au sérieux la phrase de Valéry - "Ce qu'il y a de plus profond en l'homme, c'est la peau "-et voir ce qui se passe là où la peau et la sensation et le toucher peuvent être ces comportements : frictions, caresses, limites, et toucher sans contact. [...] Comment

\footnotetext{
13. Ibid., p. 61-62.

14. Ibid., p. 121 sq.

15. Dagognet, La Peau découverte, p. 32.

16. Cf. Paul Schilder, L'Image du corps, Paris, Gallimard (coll. " Tel »), 1945, p. 107.

17. Anzieu, Moi-peau, p. 39.
} 
se crée du sens à même la peau ? [...] La question est de savoir dans quelle mesure une certaine expérience de la peau fait sens en prenant en charge ce qu'était la profondeur de la chair et dans quelle mesure elle fait sens en instaurant d'autres opérations de la sensation et du sens. La peau serait alors dans le premier cas le plus révélateur de la profondeur essentielle d'un individu, et c'est pour cela que l'on pourrait dire que " le plus profond c'est la peau ", parce qu'elle révèlerait les traits essentiels cachés dans les profondeurs subjectives. Dans le second cas, l'on pourrait dire que la peau est le plus " profond " de manière ironique, en sachant qu'elle tient par elle-même une certaine manière d'être, en contact, en caresse, en coup, en blessure, en cicatrice [...].

Explorer la peau, cette surface, en tant que telle et non comme superficie d'apparition des symptômes, écran de projections des essences bien cachées ou surface d'inscription des sens profondément ancrés. Considérer toutes choses comme des "inventions " de la peau. Explorer la peau dans son fonctionnement, et voir par exemple comment la peau peut être pensée à partir d'une série de paradoxes, de quasi-contradictions : la peau est délimitation et zone d'échange en même temps puisqu'en même temps qu'elle est une frontière de délimitation d'un corps, elle est ce qui permet l'échange ${ }^{18}$.

Nous choisirons comme corpus d'étude des œuvres de Kafka et d'actionnistes viennois, dont le rapport de filiation évident s'explique par la place centrale qu'ils réservent au corps mutilé; un corps stigmate de la violence de la norme sociale, qui annonce chez le Kafka de La Colonie pénitentiaire son instrumentalisation au service d'un arbitraire aveugle et qui, exposé, souillé, profané chez les actionnistes, marque le retour du refoulé de l'histoire et de la réification de l'homme. Nous convoquerons aussi Sylvia Plath qui, bien qu'étrangère à l'histoire autrichienne, est une héritière assumée de Kafka et de son recours au journal comme outil d'auto-analyse, comme laboratoire de l'œuvre et comme espace de jeu avec l'autofiction; nous montrerons que ces artistes explorent tous les limites du corps tout en lui réservant une place essentielle en tant que lien au monde dans la jouisssance de la caresse comme dans la blessure ou la mutilation.

\section{La peau caressée}

La caresse recrée l'être qu'elle caresse. Jean-Paul Sartre, L'Être et le Néant ${ }^{19}$

Dans L'Être et le Néant, Sartre développe une phénoménologie de la caresse, affirmant que par la caresse, nous tentons de troubler l'autre, de provoquer un bouleversement de sa conscience, en l'objectivant tout en permettant son incarnation. Il ne s'agit pas d'un simple contact entre deux êtres, mais d'une forme de communication symbolique, muette, témoignant de la conscience

18. Marie Bardet, "Ce que peut une surface ", Implications philosophiques, publié le 9 mai 2016, p. 1. Cf. www.implications-philosophiques.org/actualite/une/ce-que-peut-une-surface [dernière consultation le 18 avril 2019]. NB : c'est l'auteur qui souligne. Voir aussi à ce sujet Gilles Deleuze, Logique du sens, Paris, Minuit, 1969, p.127 et Gilbert Simondon, L'Individuation à la lumière des notions de formes et d'information, Paris, Jérôme Millon, 2005.

19. Jean-Paul Sartre, L'Être et le Néant. Essai d'ontologie phénoménologique, Paris, Gallimard (coll. " Tel »), 1943, p. 445-446. 
de l'existence de l'autre comme sujet de ses propres pensées et comme " être-pour-autrui ${ }^{20}$ ", façonné par le regard et le geste de l'autre. La caresse est ainsi une façon de s'approprier le corps de l'autre en affirmant sa propre transcendance, tout en le faisant exister comme chair au milieu du monde et des autres choses. C'est une tentative pour atteindre et "toucher" (au sens propre comme figuré) l'autre dans sa chair et dans sa conscience, qui toutes deux m'échappent par leur transcendance; caresser, c'est donc (tenter de) transcender la transcendance de l'autre :

La caresse ne se veut pas simple contact [...]. C'est que la caresse n'est pas simple affleurement : elle est façonnement. En caressant autrui, je fais naître sa chair par ma caresse, sous mes doigts la caresse est l'ensemble des cérémonies qui incarnent autrui ${ }^{21}$.

Mais cette révélation de la chair d'autrui ne peut se faire que si moi-même je me fais chair passive, objet (quasi) inanimé qui se pose tout contre l'autre et accepte sa caresse, objet à la fois touchant et touché. Je me pose alors comme conscience qui se sent exister sous le regard de l'autre, dans un processus de possession et d'incarnation réciproques :

\begin{abstract}
La révélation de la chair d'autrui se fait par ma propre chair : dans le désir et dans la caresse qui l'exprime, je m'incarne pour réaliser l'incarnation d'autrui; et la caresse, en réalisant l'incarnation de l'autre, me découvre ma propre incarnation; c'est-à-dire que je me fais chair pour entraîner l'autre à réaliser pour soi et pour moi sa propre chair et mes caresses font naître pour moi ma chair en tant qu'elle est, pour autrui, chair le faisant naître à la chair ${ }^{22}$.
\end{abstract}

Dans les récits de Kafka, la caresse manifeste la quête de contact amical ou érotique. Ainsi dans le récit de jeunesse inachevé Description d'un combat où est mise en scène l'errance nocturne dans les rues de Prague du narrateur et de son mystérieux compagnon et où le contact tactile fait figure de leitmotiv. Le narrateur évoque l'élan inattendu de son compagnon, qui se penche soudain sur un garde-fou au-dessus d'une rivière et met " son front entre ses mains ${ }^{23}$..." Mains qui semblent douées d'une vie autonome. Le compagnon voit en effet la nuit ses mains rouges s'agiter dans ses manchettes; le narrateur en est " très ému " et se met en réaction à courber l'échine et à " toucher ses genoux de ses mains en marchant ${ }^{24}$ ". Il tombe alors sur le verglas et se blesse le genou; son compagnon se penche vers lui et le " caresse doucement ", lui passant " la main sur les pommettes et deux doigts sur [son] front bas ${ }^{25}$ ". Dans le second chapitre du récit, intitulé "Chevauchée ", c'est le rapport sadique avec le compagnon qui est évoqué : le narrateur chevauche les épaules de son comparse, le tirant par les cheveux dès qu'il trébuche et lui donnant des coups sur la tête dès qu'il soupire;

20. Ibid., p. 344 .

21. Ibid., p. 430-431. C'est l'auteur qui souligne.

22. Ibid., p. 431. C'est l'auteur qui souligne.

23. Franz Kafka, Description d'un combat, Euvres Complètes II, trad. de l'allemand par Claude David, Marthe Robert et Alexandre Vialatte, Paris Gallimard (coll. " Pléiade »), 1980, p. 12.

24. Ibid., p. 10.

25. Ibid., p. 12. 
lorsqu'il tombe et est blessé au genou, son comparse l'abandonne sur les cailloux et le livre aux vautours qui, "le bec grave ", " se posent sur son corps ${ }^{26}$."

Dans La Métamorphose, récit de la " pathologie d'une peau effrayante, livrée à une agitation grouillante qui renvoie à l'image d'un corps qui ne parvient plus à se tenir dans sa propre enceinte et qui a déjà entamé un processus de décomposition ${ }^{27}$ ", Gregor Samsa tente également de trouver un réconfort érotique en collant sa peau qui le démange au portrait de La Vénus à la fourrure, atteignant ainsi un état de sérénité et d'apaisement momentané :

Il se dépêcha de monter sur la cloison et de s'appuyer sur le verre qui adhéra à son ventre brûlant et le rafraîchit d'une façon délicieuse. Cette image que Grégoire recouvrait complètement, personne au moins ne viendrait la lui prendre ${ }^{28}$.

Sylvia Plath a également fait de la peau le thème dominant de son œuvre autobiographique et fictionnel. Durant toute sa vie, elle s'est efforcée de lutter contre la souffrance d'être au monde, compensée par des moments d'extase amoureuse où la caresse du soleil et de son amant lui procurait des instants de sérénité absolue. Ainsi à l'été 1951 où la diariste évoque le contact entre sa chair et le corps du monde avec une extrême sensualité :

Couchée à plat ventre sur la roche chaude, je laissais pendre mon bras sur le côté caressant de la main les contours arrondis de la pierre bouillante sous le soleil, les ondulations lisses. Le rocher brûlant offrait un tel confort, rude et chaud, qu'on aurait dit un corps humain. [...] Le soleil s'infiltrait par chaque pore, comblait chacune des fibres douloureuses de mon corps, pour me plonger dans une quiétude dorée, rayonnante [...]. Sous moi, le corps de mon amant était chaud et pervers, la sensation de sa chair sculptée à nulle autre pareille : ni douce ni malléable, ni trempée de sueur, mais sèche et dure, lisse, propre et pure [...]. Le corps de mon amant était comme les algues, tranchant, cassant, à l'odeur puissante; comme la pierre, il était net et arrondi, incurvé ovale : comme le vent, âcre et salé [...]. C'était un sacrifice orgiaque sur un autel de pierre et de soleil $^{29}$.

La caresse ou le contact, même furtif, des peaux est donc ici gage de bien-être et de protection, remplissant cinq des huit fonctions du moi-peau relevées par Anzieu, fonctions de maintenance du psychisme, de pare-excitation ou de soutien de l'excitation sexuelle, d'individuation et enfin d'inscription des traces sensorielles tactiles.

26. Ibid., p. 17.

27. Mathias Schäfer, "Le corps garant du réel ", La Lettre de l'enfance et de l'adolescence 2007/3 ( $n^{\circ}$ 69), p. 87-96; https://www.cairn.info/revue-lettre-de-l-enfance-et-de-l-adolescence-2007-3page-87.htm [dernière consultation le 18 avril 2019], p. 184.

28. Kafka, La Métamorphose, Euvres complètes II, p. 221-222.

29. Sylvia Plath, Journaux, Euvres, éd. établie et présentée par Patricia Godi, Paris, Gallimard (coll. " Quarto »), 2011, été 1951, p. 868. 


\section{La peau marquée}

Pour une chair blessée, l'écriture est le meilleur des plans de bataille, le bon motif à imiter pour se régénérer et se retendre.

Régine Detambel ${ }^{30}$

Le revers de la caresse, soit la griffure ou la blessure, sont tout aussi présents chez Kafka que chez Plath. Le désir contenu de caresses chez Kafka est de fait proportionnel à sa détresse existentielle; le sentiment d'étrangeté aux autres et au monde dont il souffre sont liés au fait qu'il n'a qu'un " centre abstrait, aucune épaisseur charnelle et nulle peau continue à poser sur la surface du monde " et qu'il évoque un perpétuel " manque d'enveloppement ou de contenant. ${ }^{31}$ " Il souligne en effet dès les premières pages de son journal le "désespoir que [lui] cause [son] corps et l'avenir de ce corps ${ }^{32}$ "; en août 1911, il écrit avoir pour la première fois " cessé d'avoir honte de [son] $\operatorname{corps}^{33}$ " dans les piscines de Prague, Königssaal et Czernoschitz; le défaut de cette unité corporelle nommée par Lacan " stade du miroir " se manifeste par une sensation d'éclatement, d'éparpillement du corps ; d'où la sensation kafkéenne d'apesanteur qui manifeste sa " décorporéisation " s'accompagnant d'une " déréalisation par fragmentation ${ }^{34}$ " : " sans poids, sans os, sans corps, j'ai marché pendant deux heures à travers les rues ${ }^{35}$ "; d'où le sentiment " d'avoir au milieu du corps une pelote qui s'enroule très vite, tirant à elle un nombre infini de fils fixés la surface de [son] $\operatorname{corps}^{36}$ ", image que l'on retrouve dans le Souci du père de famille où la forme indéfınissable d'Odradek, sorte de bobine de fil perpétuellement en mouvement et totalement insaisissable et incompréhensible figure le corps de l'artiste. Or, la peau porte très souvent les stigmates du mal-être de ce " corps en morceaux ", perçu en rêve comme écorché, tailladé, transpercé et dont le diariste évoque la maigreur, la fragilité, les défaillances continuelles et notamment les démangeaisons :

\footnotetext{
Je suis probablement malade, depuis hier, tout le corps me démange. L'après-midi, j'avais le visage si brûlant et si diversement coloré que me faisant couper les cheveux, j'eus peur que le garçon coiffeur, qui pouvait, lui, me voir à tout instant en même temps que mon reflet dans la glace, ne constatât sur moi une grave maladie ${ }^{37}$.
}

Dans les récits de Kafka aussi, le corps est souvent blessé, pénétré, supplicié. La peau est de fait fréquemment marquée par des cicatrices : celles ramenées de la

30. Régine Detambel, Petit éloge de la peau, Paris, Folio, 2007, p. 79.

31. Jean-Marie Jadin, "La détresse de Kafka ", Cairn, p. 143-162, https:// www.cairn.info/article_p. php?ID_ARTICLE=FP_016_0143 [dernière consultation le 17 juin 2018].

32. Kafka, Journal, 1909, in Kafka, CEuvres Complètes III, trad. de l'allemand par Marthe Robert, Claude David et Jean-Pierre Danès, éd. présentée et annotée par Claude David, Paris, Gallimard (coll. "Pléiade "), 1984, p. 4.

33. Kafka, Journal, 15 août 1911, p. 35.

34. Jadin, "La détresse de Kafka ", p. 146.

35. Kafka, Journal, 6 juin 1912, p. 258.

36. Ibid., 3 novembre 1911, p. 142.

37. Ibid., 20 octobre 1911, p. 115. 
guerre par le père de Georg Bendemann dans Le Verdict, celle, devenue carapace, de Gregor Samsa bombardé de pommes par son père dans La Métamorphose, dont la blessure reste incrustée dans sa chair, entravant sa mobilité. Dans le Médecin de campagne, la contemplation de la plaie du malade constitue le point de fuite de la quête du médecin :

Dans le flanc droit à la hauteur de la hanche, une plaie grande comme une soucoupe s'est ouverte. Rose, nuancée de mille tons, sombre au fond, puis de plus en plus claire à mesure qu'on se rapproche des bords, fine de grain, avec du sang qui s'accumule irrégulièrement, ouverte comme un puits de mine [à ciel ouvert]. [...] Des vers, de la grosseur et de la longueur de mon petit doigt, roses et barbouillés de sang, se tordent $\mathrm{au}$ fond de la plaie qui les retient, pointent de petites têtes blanches et agitent à la lumière une foule de pattes minuscules. Pauvre garçon, on ne peut plus rien pour toi. J'ai découvert ta grande plaie; tu péris de cette fleur dans ton flanc ${ }^{38}$.

Cette plaie visuellement évocatrice du sexe féminin interdit, leitmotiv récurrent des écrits autobiographiques et fictionnels de Kafka, rappelle le récit de rêve du journal du 10 octobre 1911 où Kafka avait rêvé d'une fille dont le dos était couvert " de grands cercles d'un rouge de cire à cacheter dont les bords palissaient, et d'éclaboussures rouges disséminées dans les intervalles ${ }^{39}$ ".

Dans Rapport pour une académie, récit de 1917, c'est encore la cicatrice gravée sur la peau qui témoigne du lourd passé d'animal de l'ex-singe capturé et blessé par des chasseurs : " une sur la joue qui le fait surnommer Peter Le Rouge, une au-dessus de la hanche qui le fait boiter : le trou de la balle rappelle que sa nature de singe n'est pas entièrement étouffée ${ }^{40}$. "

Pour parer à cet éparpillement et à ce morcellement du corps, Kafka trouve dans la création littéraire cette seconde peau - souvent décrite à travers la métaphore de la carapace ou de la cuirasse -, ce contenant assurant maintenance $\mathrm{du}$ psychisme, individuation et prévenant l'angoisse de morcellement du corps dont parlait Anzieu; la peau du texte crée cet espace de délimitation par rapport à autrui, cette limite lui permettant d'être spectateur d'un monde dont il se sent si souvent étranger. C'est pourquoi, dans le Journal, le sacrifice du corps d'homme au travail et à la création s'exprime également métaphoriquement par la peau; le diariste compare ainsi les mots " à de la viande crue, de la viande coupée à même [sa] chair ${ }^{41}$ ".

Si Sylvia Plath évoque avec une extrême sensualité la caresse du soleil ou de ses amants sur sa peau, le leitmotiv de la démangeaison est, comme chez Kafka, très récurrent dans ses journaux. Y abondent les métaphores où la peau malade figure les déchirements intérieurs de la femme et de l'artiste; ainsi en 1952 lorsqu'elle évoque ces " émotions, putréfiées en [elle], se boursouflant et se déformant comme des plaies gonflées de pus ${ }^{42}$ "; en 1953, elle décrit la peau de sa jambe déplâtrée comme " décolorée, jaunie ", partant par " morceaux, blanche

38. Kafka, Un médecin de campagne, Euvres complètes II, p. 444.

39. Kafka, Journal, 10 octobre 1911, p. 103.

40. Kafka, Communication à une académie, CEuvres complètes II, p. 512.

41. Kafka, Journal, 3 octobre 1911, p. 91.

42. Plath, Journaux, 14 novembre 1952, p. 900. 
à vif, douloureuse ${ }^{43} \%$. Adepte du grattage, l'une des formes archaïques du retournement de l'agressivité sur le corps par la blessure délibérée " confrontant à la sollicitation de la douleur pour exister ${ }^{44}$ ", elle se griffe au sang. Témoignent de ce mal-être vécu jusque dans sa chair maints passages des Journaux où Plath évoque la texture visqueuse de sa peau :

Le moral est bas ce matin. Je n'ai pas bien dormi cette nuit. [...] Au réveil j'avais la tête lourde et l'impression de sortir d'une piscine d'eau chaude et polluée. J'avais la peau grasse, le cheveu raide et huileux, et les mains comme si j'avais touché quelque chose de sale et de visqueux [...]. J'ai le sentiment que je pourrais bien passer toute la journée à me laver dans une eau froide et pure, sans parvenir à éliminer cette pellicule sale et collante ${ }^{45}$.

\section{Ou encore :}

Mercredi 14 mai. Mauvaise nuit. La démangeaison brûlante de ma paupière s'est propagée, dans la réalité, ou par sympathie nerveuse imaginaire, à tout mon corps, du cuir chevelu aux jambes et au ventre. Comme si une démangeaison infectieuse prenait ici ou là, et me brûlait. J'ai envie de me gratter à m'en arracher la peau [...]. Moi je suis déjà partie en esprit, même si je m'attarde dans un corps de sauterelle plein de fourmillements et de démangeaisons. Je me sens devenir lépreuse ${ }^{46}$.

On songe ici aux fantasmes masochistes évoqués par Freud à propos de l'homme aux rats où l'horreur se mêle à la jouissance, celle-ci étant maximale quand le châtiment corporel - fessée, flagellation, piqûres - est appliqué à la surface de la peau, provoquant un plaisir de la trace laissée sur le corps.

Si le leitmotiv de l'écrivaine était « je suis bien dans ma peau ", lui apporte une contradiction flagrante cet autoportrait où la peau témoigne d'un dégoût de soi flagrant. Dégoût auquel, comme Kafka, seule la création lui permet d'échapper, substituant au corps de chair mal-aimé le corps d'un texte perpétuellement retravaillé :

Nez rond comme une saucisse qui coule, pores dilatés remplis de pus et de points noirs, tache rouge, et bizarre grain de beauté marron sous le menton que je regrette de ne pas avoir fait enlever [...]. Cheveux sans apprêt [...], visage sans ossature. Corps qui a besoin d'un bon lavage, la peau étant le pire à cause du climat : gerçures du froid, dessèchement de chaleur. Il faut que je sois bronzée, entièrement brunie, alors ma peau s'éclaire et ça va. Il faut que j'écrive un roman, un poème [...] et alors je serai radieuse et sans pores dilatés. Et ma verrue ne sera pas une tumeur maligne ${ }^{47}$.

43. Ibid., 20 février 1953, p. 911.

44. David Le Breton, La peau et la trace. Sur les blessures de soi, Paris, Métailié, 2003, p. 10.

45. Plath, Journaux, été 1950, p. 849.

46. Ibid., 14 mai 1958, p. 1075.

47. Ibid., janvier 1959, p. 1138. 


\section{La peau mutilée}

Les mutilations de la peau - parfois réelles, le plus souvent imaginaires - sont des tentatives dramatiques de maintenir les limites du corps et du Moi,de rétablir le sentiment d'être intact et cohésif.

Didier Anzieu ${ }^{48}$

Dans son essai intitulé "Pouvoir et $\operatorname{corps}^{49}$ ", Michel Foucault décrit le corps humain dans sa spatialité et démontre dans quelle mesure la norme s'inscrit dans le corps, parvient à pénétrer dans le corps de l'individu afin de lui imposer une courbe déterminée, de le façonner, de le contrôler, voire de le réprimer. L'État se sert en conséquence des corps de ses sujets comme prothèse pour l'exercice de son pouvoir.

Les actionnistes vont pousser à son paroxysme le thème de la blessure et de la torture présents chez Kafka, chez qui la violence de la loi sociale ou du pouvoir arbitraire s'inscrit ainsi de la manière la plus indélébile et radicale dans la peau, " enceinte infranchissable, sinon à provoquer l'horreur ${ }^{50}$ ". Dans La Colonie pénitentaire, la constante dépossession du corps s'exprime par le " fantasme d'une peau perforée ou saignante ${ }^{51}$ "; la peau marquée par la herse qui grave dans la chair du condamné les mots de la sentence témoigne du sacrifice de l'homme à la Loi toute-puissante. Le journal est perclus de nombreuses visions de l'artiste comme d'un écorché anatomique ou d'un blessé entamé par un couteau ${ }^{52}$, par des flèches comme Saint-Sébastien, par une épée ou par une machine infernale qui écrit la loi jusque dans sa chair :

Être tiré avec une corde qu'on vous a mise autour du cou, passer par la fenêtre du rezde-chaussée d'une maison et être enlevé violemment, sans ménagements, comme par quelqu'un qui ne pense pas à ce qu'il fait, être enlevé, sanglant et en lambeaux, à travers les plafonds, les meubles, les murs, les greniers, jusqu'à ce qu'apparaisse sur le toit le nœud vide qui n'a d'ailleurs perdu mes restes qu'en perçant les tuiles ${ }^{53}$.

Les actionnistes viennois font du body art une " critique par le corps des conditions d'existence [...]. Le corps est le lieu rayonnant où est questionné le monde. L'intention n'est plus l'affirmation du beau, mais la provocation de la chair, le retournement du corps, l'imposition du dégoût ou de l'horreur, le jaillissement spectaculaire du refoulé54. "Ils refusent un art bourgeois de la belle apparence trompeuse au profit d'une esthétique de la subversion et de la provocation, recourant également au support corporel pour dénoncer à travers la théâtralisation de la posture de supplicié de l'artiste la cruauté sociale et

\footnotetext{
48. Anzieu, Moi-peau, p. 42.

49. Michel Foucault, "Pouvoir et corps ", Quel corps ? 2, septembre-octobre 1975, p. 2.

50. Le Breton, La peau et la trace, p. 10.

51. Schäfer, "Le corps garant du réel ", p. 185.

52. Kafka, Journal, septembre 1915, p. 397.

53. Ibid., 21 juillet 1913, p. 302-303.

54. Le Breton, La peau et la trace, p. 100-101.
} 
les maux de l'humanité. Ainsi Günter Brus, qui se revendique comme l'héritier de Nietzsche et de Baudelaire, mais aussi de Schiele, Soutine, Picasso, Bacon et Fontana. Après avoir éprouvé pendant son service militaire le pouvoir absolu exercé sur le corps par le système répressif de l'armée, il réclame l'abolition de la domination de l'État et de ses prolongements, la famille, l'école, la police, affirme vouloir " complètement sortir de cette peau puante et pourrie " qui est la sienne et " muer absolument ${ }^{55}$ ", rejetant un art du reflet et de l'apparence au profit d'un art vivant où le corps est la " visée ", l'" événement " et le " résultat ", comme il l'affirme dès $1965^{56}$. Nourrissant l'utopie d'une humanité démocratique et paisible une fois libérée de la morale bourgeoise parce qu'assumant toutes ses pulsions, sans être aliéné par des tabous et des instincts obscurs et refoulés par le dégoût, la honte ou le péché, il représente le corps exhibé, violenté, disséqué, souillé, profané et déclare que les artistes sont "devenus des CORPORATIONS et [que] la peau d'artiste appartient à un ORDRE DU CORPS. Seules les blessures des merveilles peuvent arracher mes cicatrices ${ }^{57}$ ".

Dans l'action du 5 juillet 1965 intitulée Promenade à Vienne ${ }^{58}$, son corps est entièrement recouvert d'une peinture blanche traversée par une ligne noire, forme de recouvrement de la peau (Selbstbemalung) qui symbolise la division entre l'individu et la cité, l'artiste et le citoyen ordinaire; l'artiste dénonce par là le hiatus entre la liberté de l'art et sa répression par la société qu'incarne l'officier de police qui le verbalise et met fin à son action. Brus traverse ainsi le centre-ville de Vienne, transgressant en surface la frontière entre intérieur et extérieur du corps, entre espace artistique et espace public :

55. Arnulf et Franziska Meifert, "Günter Brus : je suis, donc j’essaie de me faire ", in Günter Brus : limite du visible, Musée national d'art moderne - centre de création industrielle, exposition au Centre Georges Pompidou, 12 octobre 1993 - 2 janvier 1994, Paris, Éditons du Centre Pompidou, 1993, p. 18. Voir aussi : Wiener Aktionismus - Brus, Günter. Malerei, Selbstbemalung, Selbstverstümmelung, Wien, Le Marais, 1965. Et Danièle Roussel, L'Actionnisme viennois et les Autrichiens, Paris, Les Presses du réel, 2008.

56. Cité par l'artiste dans : Catherine Grenier, "Interview avec Günter Brus ", in Günter Brus : limite du visible, p. 273 : " My body is the intention, my body is the event, my body is the result ".

57. Ibid., cité par Arnulf et Franziska Meifert, p. 33. NB : c'est Brus qui souligne.

58. "Im Wiener Aktionismus werden Körper und Haut instrumentalisiert und als vermittelndes Element funktionalisiert. Besonders die Haut dient dabei - als umschließende Hülle des Körpers, die im Außen stets präsent ist - der Vermittlung von Bedeutungen. [...] Dass die Haut ihrer Funktion nach als Medium charakterisiert werden kann, soll durch Günter Brus' Aktion Wiener Spaziergang von 1965 anschaulich gemacht werden. Diesem Vorgehen folgend werden zwei weitere Funktionen der Haut in Beziehung zu einer ausgewählten Aktion Brus' gesetzt. Dabei geht es zum einen um die Funktion der Haut als Passage, im Sinne der hautspezifıschen Durchlässigkeit, bei der sich das Innere im Äußeren widerspiegeln, das Äußere in das Innere dringen kann. Zum anderen wird das der Passage entgegengesetzte Phänomen der Haut als Grenze thematisiert. », in Martina Scheede, Mit Haut und Haaren. Der Körper in der zeitgenössischen Kunst, Köln, 2002, p. 55, cité dans Linda Schröer, "Hautsache Aktion”, onlinejournal kultur \&t geschlecht \#7 (2011), Cf. https://kulturundgeschlecht.blogs.ruhr-uni-bochum.de, [dernière consultation le 11 juin 2019] . Voir aussi : Kerstin Braun, Der Wiener Aktionismus. Positionen und Prinzipien, Wien, Böhlau, 1999; Thomas Dreher, Performance Art nach 1945. Aktionstheater und Intermedia, München, Fink, 2001. 
L'acte de se peindre soi-même constitue une tentative de surmonter la corporéité individuelle et ses limites : la peau de Brus est ainsi transformée en toile, le corps devient support iconographique. La peau prend donc d'abord la fonction de toile en servant de surface destinée à être peinte. Dans l'étape suivante, elle devient elle-même signe, image. ${ }^{59}$

Dans son ultime action publique, Épreuve de déchirement, le 19 juin 1970, Brus éprouve dans la profondeur la transgression de cette frontière, allant jusqu'à l'automutilation en s'attaquant à sa peau qu'il incise avec une lame de rasoir ${ }^{60}$. L'artiste poursuit ici jusqu'au simulacre du suicide le travail abordé avec Promenade à Vienne, dans une forme d'opération dramatique, s'attaquant à la peau, l'ultime frontière entre intérieur et extérieur ${ }^{61}$. Cette action est sans doute l'œuvre la plus intense de Brus, qui mène le spectateur jusqu'à un état d'extrême limite du visible et du soutenable. Avec cette action, en effet, la destruction totale du corps, évoquée par les mutilations et scarifications que l'artiste s'inflige, font désormais partie du domaine du possible. La répression que Brus subit en tant que fragment du monde social, il l'inflige à son tour à son propre corps en le torturant : le point culminant de l'action est atteint au moment où Brus se blesse la cuisse puis incise son crâne (la ligne noire peinte sur son corps dans Promenade à Vienne est ici devenue sang). Pour Brus, détruire son corps signifie l'autodestruction $d u$ monde. Mais cette destruction symbolise aussi celle qu'il subit par le monde ${ }^{62}$. Le sang, ici, est un signe de discours, il signale la violence

59. Schröer, "Hautsache Aktion", p. 7.

60. Voir, sur cette extrême limite de son art, l'entretien de Brus avec Catherine Grenier, Günter Brus, Limites $d u$ visible, p. 105-106 : "Un art qui doit aussi opérer avec le matériau provenant du monde de la cruauté pour rendre manifestes de nouvelles façons de voir n'est jamais violent, mais il est en fin de compte le seul art humain."

61. "Die Haut gilt [...] nicht nur als der Ort, da etwas Äußeres zu einem Vorgang im Innern des Menschen wird, sondern auch als Ort, da eine innere, seelische Beschaffenheit oder ein innerer seelischer Vorgang an der Oberfläche erscheint. " Cf. Ulrike Zeuch, Vermittler zwischen Innen und Außen, Haut zwischen 1500 und 1800. Verborgen im Buch, Verborgen im Körper, Wiesbaden, Harrassowitz, 2003, p. 65-84, ici p. 73.

62. Brus, Zerreißprobe, Aus unseren Protokollen; cité par Rosemarie Brucher, Durch seine Wunden sind wir geheilt, Selbstverletzung als stellvertretende Handlung in der Aktionskunst von Günter Brus, Wien, Löcker, 2008 : " Brus kniet bekleidet mit Slip, Damenstrümpfen auf einem weißen Tuch. Er legt ein durchsichtiges Plastikdreieck auf seinen Schenkel und schneidet an einer Kante mit einer Rasierklinge ins Fleisch. Brus klappt das Dreieck zum Knie und wartet, bis das Blut am Dreieck hinunterläuft. Blitzschnelles Wälzen am Boden. Brus hakt zwei Schnüre neben der Wunde in den Strumpf und klafft ihn auseinander: Dann steht er auf und sagt mit ruhiger Stimme: 'Kann mir jemand ein Glas hergeben?' Brus uriniert in das Glas und trinkt es aus. Er schneidet sich mit einer Schere Strümpfe und Hose auf. Brus steht nackt mit dem Rücken zu den Leuten und rutscht angespannt an der Wand herunter bis er kniet. Er schneidet sich mit der Rasierklinge in den kahlen Schädel und wartet, bis aus dem Schnitt das Blut bis zum Gesäß läuft. Er bindet Schnüre um seine Knöchel und zieht mit den Schnüren die Beine auseinander, dabei rutscht er an der Heizung zu Boden. Er sagt mit ruhiger Stimme: 'Ich hätt' noch gern ein Glas'. Er schreit: 'Nein! Nein!' Blitzschnelles Wälzen am Boden. Er steigt mit den Füssen in kleine rechteckige Wannen voll Wasser, rutscht aus, steigt in die nächste. Mit ruhiger Stimme sagt er: 'Kann mannicht das Fenster schließen?', wartet aber keine Reaktion ab, sondern wirft sich zu Boden, peitscht mit einem Riemen auf den Boden, ins Wasser, schreit, wälzt sich bis zur Erschöpfung, wölbt den Bauch nach oben, berührt den Boden nur mit Kopf und Füssen, verharrt so bis zur Erschöpfung. Brus geht durch die Zuschauer zur Toilette. Ende der Aktion. » 
exercée par l'État et la société63 sur le corps de l'artiste qui déclare : « L'État veut me manger, rôtir, sucer, baiser, congeler, décongeler, inventer ${ }^{64}$. " Et au corps symboliquement sacrifié sur l'autel de la Loi fait miroir le corps virtuellement supplicié de l'artiste :

Je coupe ma main gauche. Quelque part se trouve un pied. Une suture sur le poignet. J'enfonce une punaise dans la moelle épinière. Je cloue le gros orteil sur l'index [...]. À l'aide d'une lame de rasoir (de la marque Smart) j'égratigne l'artère carotide sur toute sa longueur. J'enfonce un clou dans l'oreille. Je fends ma tête sur toute sa longueur en deux moitiés. J'introduis du fil barbelé dans mon urètre et essaie doucement, en tournant, de déchirer le nerf (autocystoscopie) ${ }^{65}$.

Si les blessures de Brus sont réelles et faites en présence d'un public, celles de Schwarzkogler sont fantasmées et médiatisées par l'image. L'artiste explore également la peau en tant que zone-limite entre l'homme et le monde, en recourant au medium photographique comme mode de suggestion de blessures purement virtuelles et comme producteur d'un choc cathartique de la violence sociale :

Pour le regardeur, l'image photographique signifie un document - " fiable " - qui lui permet de croire aux cruautés et aux blessures, d'autant plus que la confrontation à cette image produit un choc visuel et émotionnel qui empêche une pensée critique [...]. La suggestion d'une image ne fait pas appel à la raison, mais aux sens, ce qui lui donne un grand pouvoir permettant d'influencer l'inconscient. [...] Il s'instaure un échange entre ce qui est montré et ce que le regardeur pense. Cette interaction crée une tension qui peut le transporter à un autre niveau de signification. L'imaginaire naît en effet de cette tension, de la distance entre le visible et l'intouchable [...]. La violence de l'image - " document " contemplée - aura toujours ce pouvoir de l'indice qui fait croire à l'existence des faits présentés, nous rendant complices - passifs - de ces excès ${ }^{66}$.

Dans l'Action $N^{\circ} 3$, réalisée à l'été 1965 , il fait poser devant un objectif son ami Heinz Cibulka ${ }^{67}$ dont le pénis entouré d'une gaze et semble entaillé par des ciseaux et des scalpels évoquant une castration symbolique et suggérant à l'imaginaire du spectateur toute l'horreur de la mutilation. Ambitionnant de devenir le Van Gogh de l'art corporel, l'artiste entraîne son public jusqu'à la limite du représentable, tant la violence de l'image suggérée rend le spectateur complice de la mutilation dont l'homme souffre dans la société :

63. Schröer, “Hautsache Aktion”, p. 15-16: «Mit der einhergehenden Inszenierung der Haut als Passage, bestätigt er, dass Haut nicht als reine Grenzfläche verstanden werden kann, sondern im beständigen Austausch mit der Umwelt seine Durchlässigkeit preisgibt. Es gelingt ihm dadurch, darauf aufmerksam zu machen, dass die Vorstellung vom Körper als glatte Fläche, als etwas Begrenztes und rein äußerlich erfahrbares, eine nicht haltbare Konstruktion der Gesellschaft zur Herstellung von Ordnung darstellt."

64. Günter Brus (dir.), " Der Staat ", Patent Urinoir, Wien, autopublié par l'artiste, 1968. Cité par Schäfer, "Le corps garant ", p. 94.

65. Brus, Auszug aus seiner Selbstverstümmelung, Wien, Le marais, 1965. NB : C'est nous qui traduisons.

66. Schäfer, " Le corps garant », p. 95-96.

67. Cf. Heinz Cibulka, Mein Körper bei Aktionen von Nitsch und Schwarzkogler 1965-1975, Napoli, Editioni Morra, 1977, et Eva Badura-Triska, Hubert Klocker, Rudolf Schwarzkogler, Kat. Museum moderner Kunst Stiftung Ludwig Wien im Museum des 20. Jahrhunderts, Klagenfurt, Ritter Verlag, 1992. 
Nous pouvons comprendre les actions à la fois comme une destruction par le monde (que l'individu/corps subit). À travers l'identité du mutilé et du mutilant, la différence entre paraître et être, imagination et réalité se trouvent annulées. Par l'intermédiaire du corps, l'art accède à la réalité. Le corps devient lieu de vérité, car la douleur ne ment pas ${ }^{68}$.

Ainsi la peau n'est-elle nullement une simple surface, mais une limite productrice de sens, comme le conclut Marie Bardet qui propose avec Gilbert Simondon une " topologie de la limite ". Simondon affirme en effet que la limite entre l'intériorité de l'individu et le milieu extérieur est toujours " relation, actuelle ou potentielle. " Loin de le délimiter comme un " domaine fermé " distinct du monde, la limite et par conséquent la relation de l'individu, n'est jamais une borne; elle fait partie de l'être même ${ }^{69}$. Reprenant à son compte cette réflexion en l'approfondissant, Bardet affirme que

penser la peau comme cette limite topologique permet de comprendre en quoi elle n'a ni direction organique ni centre de l'organe; et comment elle est limite ET relation, et non le résultat d'une intériorité ou extériorité a priori [...] Autrement dit, penser, avec Simondon, la peau comme limite topologique, c'est-à-dire relation, ce n'est pas penser les bordures de chaque chose mais chevaucher la limite et voir où elle nous mène ${ }^{70}$.

Ainsi notre analyse espère-t-elle également avoir montré que la peau, "surface d'inscription et d'interprétation du monde ${ }^{71}$ ", " milieu d'autres milieux faisant fusionner l'opacité de l'épaisseur et la visibilité de la surface ${ }^{72}$ ", nous invite à dépasser l'opposition désormais caduque entre surface et profondeur, entre extériorité et intériorité au profit d'une " esthétique de la liminalité ${ }^{73}$ " où la limite se fait productrice d'un sens jamais acquis mais toujours renouvelé, réinterrogé à chaque nouveau contact entre la peau et le monde naturel, humain et social :

Entre le dehors et le dedans, elle établit le passage des stimulations et du sens. Instance de séparation, elle enclôt l'individualité mais elle est simultanément lieu d'échange avec le monde [...]. Lieu de la limite et simultanément de l'ouverture, elle indique à l'individu sa souveraineté sur le monde, le volume qu'il y occupe ${ }^{74}$.

68. Schäfer, "Le corps garant ", p. 96.

69. Gilbert Simondon, L'Individuation à la lumière des notions de forme et d'information, Paris, J. Milon, 2005, p. 127-128.

70. Bardet, "Ce que peut une surface ", p. 17.

71. Stéphane Dumas, Les Peaux créatrices, esthétique de la sécrétion, Paris, Klincksieck, 2014, p. 19.

72. Ibid., p. 29.

73. Ibid.

74. Ibid., p. 179. 